\title{
The Effects of Optimized Regenerator Allocation in Trans- lucent Networks under Inaccurate Physical information
}

\author{
E. Marín-Tordera ${ }^{1}$, M. Yannuzzi ${ }^{1}, X$. Masip-Bruin $^{1}$, \\ S. Sánchez-López ${ }^{1}$, R. Martínez ${ }^{2}$, R. Muñoz ${ }^{2}$, R. Casellas², G. Maier ${ }^{3}$ \\ ${ }^{1}$ Advanced Network Architectures Lab (CRAAX), Technical University of Catalonia, \\ ${ }^{2}$ Centre Tecnologic de Telecomunicacions de Catalunya (CTTC) \\ ${ }^{3}$ Politecnico di Milano
}

\begin{abstract}
Optimized regenerator allocation techniques select which of the already installed regenerators in a translucent network must be used in order to maximize the quality of the optical signal while minimizing the opaqueness of the network. Unfortunately, the performance of an optimized regenerator allocation strategy strongly depends on the accuracy of the physical-layer information. In this paper, we investigate the effects of optimized regenerator allocation techniques when the physical-layer information is inaccurate. According to the performed experiments, we conclude that mostly of the current techniques of regenerator usage optimization are only possible when perfect knowledge of physical information is available. Hence, new regenerator allocation schemes taking into account the inherent inaccuracy in the physical-layer information need to be designed.
\end{abstract}

\section{INTRODUCTION}

In the last years, a critical point in the evolution of optical networks has been the increase in transmission capacity, going from bit rates of $10 \mathrm{Gbps}$ to $40 \mathrm{Gbps}$. In addition, optical networks are evolving from opaque to all-optical, i.e., transparent networks. Unlike transparent networks, in a traditional opaque network, an optical-electronic-optical (OEO) conversion is necessary at every switching node. The OEO conversion enables re-amplifying, re-shaping, and retiming (i.e., 3R regeneration) the optical signal. As a result, the signal regains the same physical features as it has at the source node. Nowadays, $3 \mathrm{R}$ regeneration is only possible by means of OEO conversion (though it is expected to be available within the optical domain in the near future), making the overall regeneration process slow and expensive.

On the other hand, in an all-optical network, the optical signal reaches the destination node without using OEO conversion at the intermediate nodes along the path. However, the maximum transmission distance that an optical signal can reach without $3 \mathrm{R}$ regeneration is limited by the physical-layer impairments (PLIs). The accumulation of PLIs degrades the optical signal quality, at the extent that the latter can become illegible at the destination. In this regard, the higher the transmission rate the higher the PLI effects are-actually, most of the PLIs have a higher effect when the network operates at rates equal or above 40 Gbps [1]. This represents a major concern, since reaching higher

\footnotetext{
* This work was partially supported by the Spanish Ministry of Science and Innovation under contracts TEC2008-02552-E and TEC2009-07041 as well as by the Catalan Government under contract 2009 SGR1508
}

transmission capacities is expected as part of the natural evolution of the optical networks.

An interim solution is provided by translucent optical networks, in which selected network nodes are equipped with $3 \mathrm{R}$ regeneration capabilities. Then the lightpaths are divided into transparent sub-paths (i.e., transparent segment between two $3 \mathrm{R}$ regenerators). It is worth highlighting that the use of $3 \mathrm{R}$ regenerator also allows the wavelength conversion capability. This leads to attain a good trade-off between network cost and end-to-end optical signal quality.

In this scenario, new RWA algorithms, so-called Impairment Aware RWA algorithms (IA-RWA), have been proposed in the literature for transparent and translucent optical networks. In the IA-RWA algorithms, the PLIs are modeled as constraints to be considered by the path computation process when selecting the route and the wavelength for a lightpath request. A summary of the most significant IARWA algorithms can be found in [2].

There are several types of PLIs, which can be grouped into two categories: linear and non-linear, each degrading the quality of the optical signal in different ways. Recent efforts have been dedicated to modeling the PLIs, with the end of making easier the study of their impact on the network performance. A PLI model must be simple enough to be useful in practice, where a limited number of input parameters should be sufficient to characterize each optical link. It is also desirable to have a single output or performance parameter. This parameter allows capturing the effects of all PLIs on each optical circuit, in order to evaluate the optical signal quality. This single output parameter collecting all the PLI effects is commonly called quality factor parameter $Q$ [3] [4] [5]. In this paper, we consider IA-RWA algorithms relying on the $Q$ Personick's factor [3], as the optical signal performance parameter.

In the context of network planning, off-line IA-RWA algorithms are used in order to calculate and allocate the necessary resources (i.e., the number of wavelengths on each link) so as to establish an estimated number of permanent connections. Additionally, in translucent networks, these IA-RWA algorithms are enhanced for computing the number of regenerators needed at each network node. This problem is referred to as the regenerator placement.

In this work, we deal with IA-RWA algorithms during the regular operation of a translucent network, that is, under dynamic traffic conditions. In such a scenario, network re- 
sources, including the $3 \mathrm{R}$ regenerators, are already placed. Upon receiving a connection request, the IA-RWA algorithm performs an on-line route computation and wavelength selection. Moreover, the IA-RWA algorithm decides whether to use or not the regenerators already placed along the nodes of the selected path; this is known as regenerator allocation.

Few works in the recent literature address the problem of regenerator placement and allocation. Moreover, these works only consider the placement/allocation based on accurate physical-layer (e.g., $Q$ ) information.

In this paper, we investigate the effects of optimized regenerator allocation techniques when the physical information used to compute the $Q$ factor is not completely accurate. In such cases, the IA-RWA process might end up selecting routes and wavelengths through which lightpaths cannot be successfully established, due to the possible discrepancy between the estimated $Q$ factor and the real one in the network. The establishment of a lightpath may also fail due to the inappropriate allocation of regenerators along the path. In this context, we evaluate an IA-RWA algorithm (MINCOD-Q [6]) combined with two regenerator allocation techniques: 1) an optimized scheme allocating regenerator resources at the nodes where the signal quality falls below a threshold; 2) a non-optimal one allocating all the regenerator resources found in the nodes along the path regardless of the optical signal quality. Our results show that, in the presence of inaccurate information, the performance of an optimized regenerator allocation technique drops sharply, indicating that such a strategy results adequate as long as accurate physical-layer information is available.

The remainder of this paper is organized as follows. Section II overviews some IA-RWA algorithms, utilizing regenerator placement or allocation techniques. Section III briefly reviews the $Q$ Personick's methodology and presents the problem of the inaccuracy in the physical information. Section IV describes the IA-RWA algorithm utilized to evaluate the effect of having inaccurate information, as well as the two different regenerator allocation techniques. Section V evaluates the performance of the regenerator allocation techniques in the presence of uncertainties; and finally, Section VI concludes the paper.

\section{IA-RWA Algorithms WITH REgENERATOR PlaCE- MENT AND ALLOCATION IN TRANSLUCENT NETWORKS}

In translucent networks, IA-RWA algorithms are usually combined with both regenerator placement and allocation techniques. The regenerator placement is part of the planning phase, in which the goal is the design of the most costefficient network which minimizes the number of regenerator resources. This is a complex optimization problem and, in particular, is solved by the use of off-line algorithms. The regenerator placement problem is addressed in [5][7][8].

Once regenerators are placed in selected nodes of the network, the regenerator allocation problem aims at minimizing/optimizing the utilization of such already installed regenerators. This problem involves the routing of the connec- tion requests to attain an efficient utilization of the network resources (i.e., wavelengths and $3 \mathrm{R}$ regenerators). To this end, several IA-RWA algorithms have been proposed in the literature, which can be classified into two categories depending on the constraints utilized to address the optical signal quality: i) algorithms which do not exceed a maximum transparent distance, otherwise regeneration is needed; and ii) IA-RWA algorithms that consider analytical expressions for particular physical impairments, such as the optical signal noise ratio (OSNR), the bit error rate (BER), the $Q$ factor, etc. In the first category, [9] proposes the 2 Dimensional (2D) algorithm, which is an-online IA-RWA mechanism that computes the shortest path not exceeding the regeneration distance limits. In the second category, [7] proposes an IA-RWA algorithm that considers the OSNR, the polarization mode dispersion, the chromatic dispersion and crosstalk as the optical constraints for the regenerator allocation. In [10], the authors present an on-line OSNR-based IARWA algorithm deployed in a GMPLS-enabled network, which considers the received OSNR level as the optical signal performance parameter. Accordingly, if this value is below a given OSNR threshold, the IA-RWA algorithm dynamically allocates available $3 R$ regenerators along the computed path. Likewise, in [11], the DWP (Distributed Discovery of Wavelengths Paths) method is presented, in which one of the objectives is the minimum usage of electronic regeneration.

\section{Q PERSONICK'S FACTOR AND UNCERTAINTIES IN THE ESTIMATION OF THE Q FACTOR}

The BER is considered as the main performance parameter to measure the optical signal quality at the receiver of an optical connection. This parameter can be ascertained taking into account the network topology, the path and, the wavelengths assigned by the IA-RWA algorithm to each traversed line system, and the allocation of regenerators. By doing so, the optical signal degradation along that lightpath can be estimated by means of the BER level. Indeed, the estimated BER has to be above a given threshold to ensure that the optical signal quality is acceptable. For this reason, an effective and simple method is required in order to estimate the optical signal degradation along a path. This can be tackled by observing that the BER threshold can be translated into a threshold value of the so-called Personick $Q$ factor [3]. The $Q$ factor can then be evaluated as a function of the transmission-system parameters,(i.e. optical bandwidth, electrical bandwidth, power level at the signal launch, etc) and the transmission impairments (ASE-noise, PMD, etc). Without any error correction mechanism on the digital signal at the receiver, a quality factor $Q=16.9 \mathrm{~dB}$ corresponds to a BER of approximately $1 \times 10^{-12}$. Typically, the requirements for the minimum value of the signal $Q$ at the receiver are about $17 \mathrm{~dB}$ without error correction, and $12 \mathrm{~dB}$ in case of error correction. The expression used for evaluating the $Q$ factor at the receiver of a transparent path (or subpath) is given by (1). 


$$
Q_{\text {end }}=a_{0}+a_{1} O S N R_{\text {end }}+a_{2} N_{S P A N}+a_{3}\left(P_{0} N_{S P A N}\right)^{B}
$$

According to (1) the quality factor depends on both linear and non-linear effects. The $O S N R_{\text {end }}$ factor is the optical signal to noise ratio expressed in $\mathrm{dB}$ at the receiver. The terms $a_{2} N_{S P A N}$ and $a_{3}\left(P_{0} N_{S P A N}\right)^{\mathrm{B}}$ take into account the nonlinear effects, considering all the amplifiers along a path (booster, pre- and line amplifiers). $N_{S P A N}$ is the number of spans forming the transparent path (a span is the segment of a link between two amplifiers). $P_{0}[\mathrm{dBm}]$ is the power level at the signal transmission (typically $3 \mathrm{dBm}$ ). The coefficients $a_{0}, a_{1}, a_{2}, a_{3}$, and $B$, depend on the type of used line systems, and should be tuned by an on-field measurement campaign [12].

However, as it is argued in [13][14][15] and [16], the physical parameters stored at the source nodes' databases, and/or the $Q$ values computed by means of the corresponding $Q$ model, may be different from the corresponding real physical values. In general, the differences between the real $Q$ values and the computed $Q$ values are due to two reasons, namely, uncertainties derived from the inaccuracy of the $Q$ model (i.e. the $Q$ Personick's model does not take into account physical impairments that depend on the traffic load); and also uncertainties due to the inaccuracy of the monitored physical parameters. The effect of these sources of uncertainty is the drift suffered by both the physical-layer parameters and the $Q$ values from their nominal values during the operation of the optical network. In these conditions, the performance of the IA-RWA process might drop sharply when either assigning routes and wavelengths or when allocating regenerators, since the real $Q$ value differs from the $Q$ factor used by the IA-RWA algorithms.

\section{OVERVIEW OF IA-RWA ALGORITHMS WITH AND WITH- OUT REGENERATOR ALLOCATION}

In light of the above, our goal is to analyze the effects of using optimized regeneration allocation techniques when the physical information is not completely accurate. To this end, we evaluate an IA-RWA algorithm: MINCOD-Q [6]. For the sake of clarification, we assume that a regenerator placement algorithm was previously used to place the regenerators according to a static traffic matrix [12].

\section{A. MINCOD-Q IA-RWA Algorithm}

The so-called Minimum Coincidence and Distance according to the $Q$ factor (MINCOD-Q) [6] is based on the Minimum Coincidence and Distance (MINCOD) routing algorithm [17]. MINCOD-Q uses a set of $K$ pre-computed routes, from a source node to a destination node, having the minimum distance in terms of kilometers and the minimum number of shared links. The $Q$ factor is computed according to the expression (1), and is used as the performance indicator to decide whether a lightpath or the set of transparent sub-paths satisfy a given $Q$-threshold constraint. For each sub-path, the selected wavelength channel is the first one available on each link forming the sub-paths because we assume wavelength conversion capability of the $3 R$ regenerators.

In short, the MINCOD-Q algorithm selects, amongst $K$ pre-computed routes, a path and the wavelength channels that allow fulfilling both the WCC (wavelength continuity constraint) and the optical signal quality restriction in each sub-path. This algorithm always allocates the available preplanned placed regenerators along the path regardless of whether the regenerator is needed to fulfill the adequate optical signal quality requirements.

\section{B. Optimization of Regenerator usage: MINCOD-Q-REG Algorithm}

If we assume that only some nodes of the network are equipped with regeneration capability, the MINCOD-Q algorithm sets up a connection allocating an available regenerator on all the intermediate nodes equipped with regenerators forming the route.

In [6], the MINCOD-Q routing decision is enhanced in order to avoid using regenerators on a node as long as it is not necessary. As a result, the new proposed MINCOD-Q-REG algorithm computes the path without using regenerators. In the first node of such a path wherein the $Q$ factor level is below the $Q$ threshold, the algorithm stops. Then, the algorithm looks for a regenerator in the previous node. If a free regenerator is found in that node, and the $Q$ factor level on that node is above the threshold, then an available regenerator is allocated. This causes that the provisional path is divided into two transparent sub-paths or segments. Otherwise, if no free regenerators can be allocated in the previous node, the algorithm seeks for a regenerator in the next previous node, and so on. This enhanced algorithm is named MINCOD-Q-REG algorithm. Results in [6] show the significant improvement of this optimization in terms of the resource consumption. With approximately only $10 \%$ of the installed regenerators, MINCOD-Q-REG behaves similar than MINCOD-Q in terms of blocking probability. However, the MINCOD-Q-REG algorithm was proposed to optimize the regenerator usage by using accurate $Q$ information. In this paper, we evaluate the potential benefits of the regenerator allocation used in MINCOD-Q-REG in the presence of uncertainties in $Q$.

\section{Performance evaluation}

Evaluation trials are performed on the Pan European network formed by 28 nodes and 41 links. All the links are equipped with systems of 40 wavelengths. The number of systems (40 wavelengths) installed in parallel on each link is calculated in a previous planning phase. On the basis of a uniform static matrix, this previous design phase give us 129 regenerators distributed in 19 of the nodes, and 56 systems distributed in the 41 links. More detailed information about the planning phase can be found in [12]. Once the network is deployed, we carry out a set of simulations under dynamic traffic conditions: from 0.1 to 1 Erlang between each pair of nodes of the network The $Q$ threshold utilized in all the fol- 


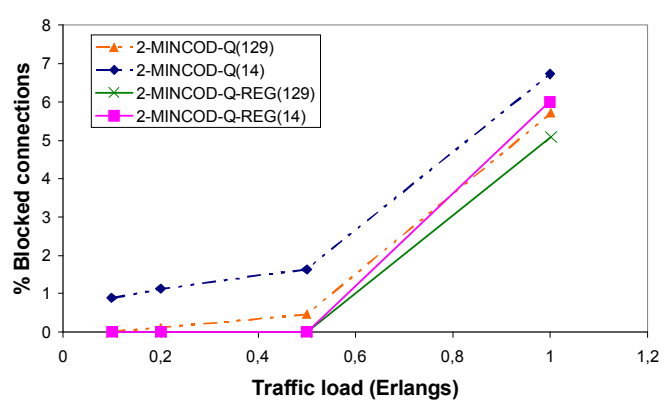

a)

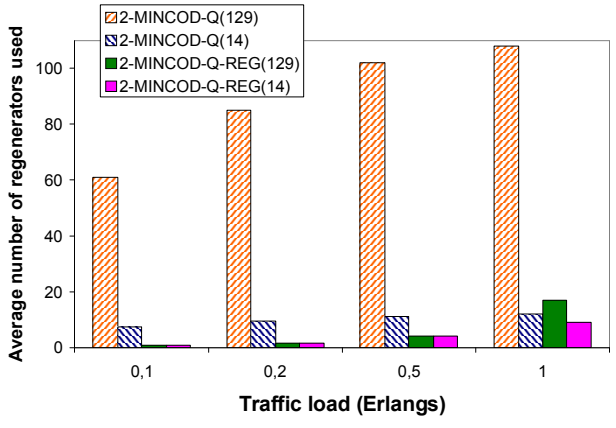

b)

Figure 1. Results of MINCOD-Q and MINCOD-Q-REG for accurate $Q$ information

lowing simulations is $17 \mathrm{~dB}$, because it approximately corresponds to a BER of $1 \times 10^{-12}$.

\section{A. Results for accurate $Q$ information}

Results depicted in Figure 1 show the percentage of blocked connections (Figure 1 a)) and the average number of used regenerators per time unit (Figure $1 \mathrm{~b}$ )) versus the offered traffic load for both MINCOD-Q and MINCOD-QREG strategies. Moreover, we also present results when the number of installed regenerators is reduced from 129 to 14 . These results correspond to the case when the information about the parameters used to compute the $Q$ factor is completely accurate. We observe that MINCOD-Q-REG, with a significant reduction of the number of regenerators, obtains practically the same blocking probability than MINCOD-Q.

\section{B. Results for inaccurate $Q$ information}

In the following set of simulations we add a degree of inaccuracy to the $Q$ factor computed by means of the $Q$ Personcik's methodology.

We analyze the impact of inaccurate physical information [15] in terms of the blocking probability when the two proposed regenerator schemes are used (that is, always using the available regenerators found along the path or only if needed).

As it is exposed previously, the $Q$ values computed by means of the $Q$ Personick's methodology may be different from the corresponding real values. In our study the discrepancies between both the computed and the real $Q$ values will be quantified in a single error value that is unknown by the IA-RWA algorithm. We then associate an error value to each one of the candidate transparent lightpaths between nodes with $3 \mathrm{R}$ regenerators. We consider that the real $Q$ value of a lightpath, which is denoted by $Q_{\text {real }}$, is the $Q$ value obtained using the $Q$ Personick's methodology, which is denoted by $Q_{e}(Q$ Personick's $)$, minus an error value:

$$
\left.Q_{\text {real }}(\text { lightpath })=Q_{e}(Q \text { Personick's }) \text { - error (lightpath }\right)
$$

This error value is a fixed amount of decibels ranged in the different set of simulations between 0 and $2 \mathrm{dBs}$; and in this study we only consider the case of underestimation of the $Q$ value. This approach of creating mismatching by a fixed amount of decibels of degradation on each sub-path is simple but effective in producing a degradation of the network blocking performance. Obviously, we assume that this error value is unknown to the IA-RWA algorithm and, as a result, the algorithm may make a wrong routing and wavelength assignment and/or a wrong regenerator allocation decision.

Figure 2 shows the percentage of blocked connections of both MINCOD-Q and MINCOD-Q-REG versus the degree of inaccuracy, i.e., error in (2) for different traffic loads. Note that an inaccuracy degree of $0 \mathrm{dBs}$ corresponds to have accurate $Q$ information. We observe that for $0 \mathrm{dBs}$ (accurate information), MINCOD-Q-REG outperforms the MINCOD$\mathrm{Q}$ performance for all traffic loads. With inaccurate information MINCOD-Q-REG only outperforms MINCOD-Q for 0.5 Erlangs with an inaccuracy of $0.25 \mathrm{dBs}$; and for 1 Erlang for an inaccuracy lower or equal to $0.5 \mathrm{dBs}$.

We can conclude that the MINCOD-Q-REG algorithm (which makes optimization of the regenerator usage) is more affected when increasing the degree of inaccuracy compared with MINCOD-Q. This is rooted on the fact that the MINCOD-Q-REG allocates regenerators only when the $Q$ factor value in an intermediate node along the path falls below a threshold. Thereby, when establishing a new lightpath, MINCOD-Q-REG can decide not to allocate a new regenerator in an intermediate node because the computed $Q$ is above the required $Q$ threshold. However, the real $Q$ value may be below that $Q$ threshold. In this situation, the lightpath will be selected without allocating a regenerator in the mentioned node. In consequence, the lightpath will be blocked in a subsequent intermediate node since the received optical signal does not have sufficient quality. On the other hand, the MINCOD-Q algorithm, which does not perform any regenerator usage optimization, in the same case, would allocate the regenerator in that intermediate node. Recall that this strategy always allocates an available regenerator at each traversed node with $3 \mathrm{R}$ capabilities along the route. Then, the selected path by MINCOD-Q lowers the blocking due to the inadequate level of the optical signal quality.

From Figure 2 we can conclude that the regenerator allocation optimization is only fruitful in the MINCOD-Q algorithm in terms of blocking probability when the degree of inaccuracy (error) is at most $0.5 \mathrm{dBs}$. This conclusion should not be extrapolated to different IA-RWA algorithms, but it is expected to have the same problem with other IA-RWA algorithms. 
129 regenerators- 0,1 Erlangs

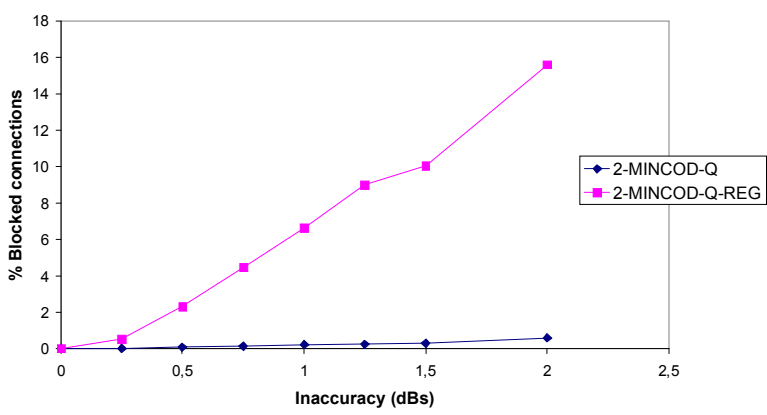

a)

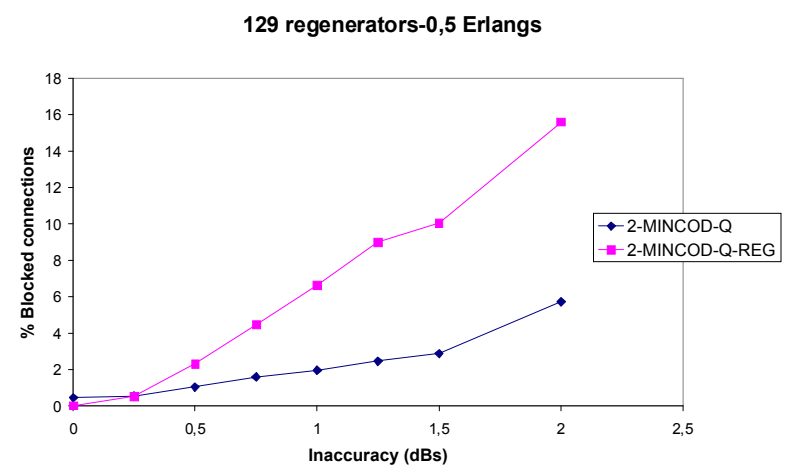

c)
129 regenerators-0,2 Erlangs

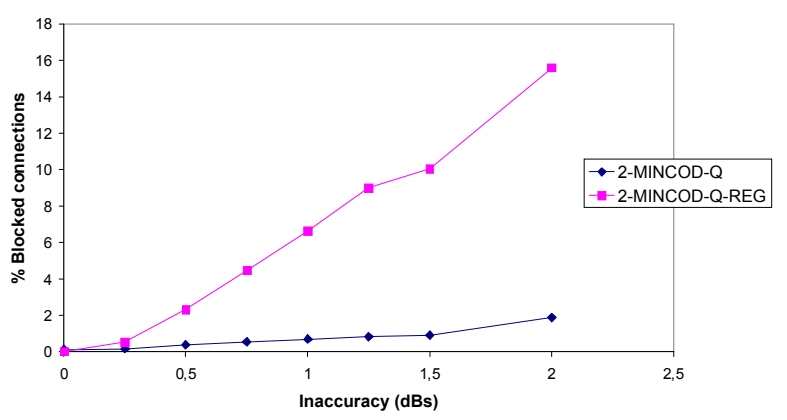

b)

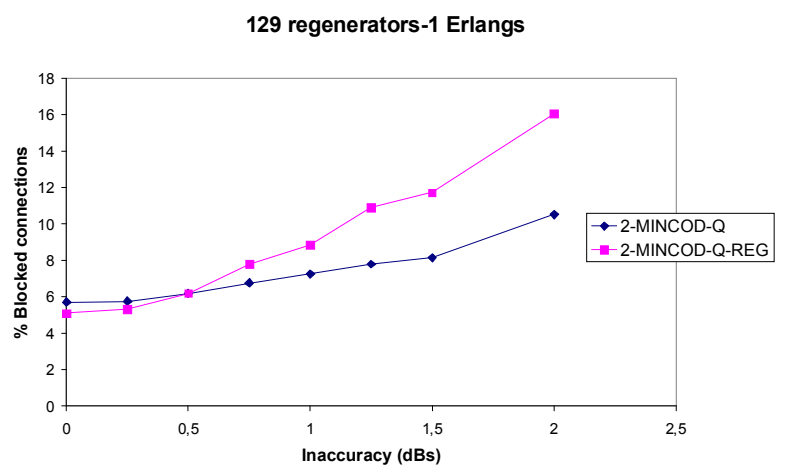

d)

Figure 2. Percentage of blocked connections versus degree of inaccuracy

In order to further evaluate the performance of these algorithms in presence of the inaccuracy of the physical information, we have carried out a different set of simulations reducing the number of installed regenerators in the network from 129 to 14 . Figure 3 shows the percentage of the connection blocking (a), and the average number of regenerators per time unit (b), versus the traffic load for both MINCOD-Q and MINCOD-Q-REG strategies considering 129 and 14 regenerators installed through the network and an inaccuracy degree set to $0.25 \mathrm{dBs}$. Figure $3 \mathrm{c}$ ) and d) show the same results when the inaccuracy degree is $0.5 \mathrm{dBs}$. From Figure 3 a) and c) and comparing them with Figure 1 a) we observe:

1. MINCOD-Q-REG scheme is highly affected by the inaccurate information to estimate the $Q$ factor. Indeed, it degrades the performance more significantly compared to the MINCOD-Q strategy;

2. The performance attained by the MINCOD-Q-REG scheme is less affected by the reduction of installed regenerators.

On the other hand in terms of regenerator consumption (see Figure $3 \mathrm{~b}$ ) and d)), the MINCOD-Q-REG outperforms the results obtained by the MINCOD-Q, needing approximately only a $10 \%$ of the regenerators that MINCOD-Q requires. In light of the numerical results, we conclude that when the degree of inaccuracy is below $0.5 \mathrm{dBs}$, we can achieve by means of regenerator allocation techniques (e.g. MINCOD-Q-REG) a significant reduction in the needed regenerators in a translucent networks in return for a slightly increase of the blocking probability. Whereas when the degree of inaccuracy is higher, regenerator allocation optimizations are not useful due to their high cost in terms of blocking probability.

\section{CONCLUSIONS}

In this paper, we show the high impact on the network performance of optimized regenerator allocation techniques when the physical-layer information is not completely accurate. We have evaluated the MINCOD-Q IA-RWA algorithm without regenerator usage optimization as well as combined with an optimized regenerator allocation scheme in the presence of different degrees of inaccuracy. The physical impairments affecting the optical signal are modeled by means of the $Q$ Personick's methodology. The degree of inaccuracy is introduced by means of a fixed quantity, which makes the computed $Q$ lower than the real $Q$. This difference between the computed and the real $Q$ factors derives in significant performance degradation.

From the results obtained, we conclude that optimizing the regenerator usage in MINCOD-Q is suitable in terms of resource consumption with either accurate information, or with a degree of inaccuracy lower than $0.5 \mathrm{dBs}$. With a degree of inaccuracy lower than $0.5 \mathrm{dBs}$, with approximately only $10 \%$ of the regenerators, the IA-RWA algorithm optimizing the regenerator usage achieves similar performance in terms of blocking probability than that of a non-optimized algorithm. However, when the degree of inaccuracy is higher, it is not advisable to use the optimized regenerator technique, because the blocking probability increases significantly. It is preferable to use a simpler IA-RWA algorithm, 
not optimized, using all the regenerators available in the path; even if it has a higher cost in terms of regenerator consumption.

New IA-RWA algorithms and regenerator allocation schemes, taking into account the inherent and unknown inaccuracy in the physical information have to be developed. A first approach can be to model or estimate the degree of inaccuracy in the computed $Q$. Then, the IA-RWA algorithms and regenerator allocation schemes could use it in order to perform a better lightpath and regenerator selection.

\section{REFERENCES}

[1] H. Büllow, J. Dobovan, R, Herschel, "Outage of 40 Gbps Optical Paths Routed over PMD-Impaired Fiber Links", OFC 2008 , San Diego California, February 2008.

[2] S. Azodolmolky, M. Klinkowski, E. Marin, D. Careglio, J. Sole Pareta, and I. Tomkos, "A Survey on Physical Layer Impairments Aware Routing and Wavelength Assignment Algorithms in Optical Networks", Computer Networks, Elsevier, ISSN: 1389-1286, Vol. 53, issue 7, May 2009.

[3] S.D. Personick, "Receiver Design for Digital Fiber Optic Communication Systems, I", Bell Syst. Tech. J, Vol. 52, No. 6, July-August, 1973, pp. 843-874.

[4] P. Kulkarni, A. Tzanakaki, C.M. Machuka and I. Tomkos, "Benefits of Q-factor based routing in WDM metro networks", ECOC 2005, Glasgow, Scotland, September 2005.

[5] M. Ali Ezzahdi, S. Al Zahr, M. Koubaa, N. Puech, and M. Gagnaire, "LERP: a Quality of Transmission Dependent Heuristic for Routing and Wavelength Assignment in Hybrid WDM Networks," Proc. of ICCCN 2006, pp. 125-136, Arlington, TX, USA, October 2006.

[6] E. Marin-Tordera, J. Solé-Pareta, R. Martinez, R. Muñoz, R. Casellas, "Improving IA-RWA algorithms in translucent networks by regenerator allocation", ICTON 2009, Azores, Portugal, June 2009.

[7] X. Yang, and B. Ramamurthy, "Dynamic Routing in Translucent WDM optical Networks the intradomain case," Journal of Lightwave

\section{Inaccuracy $0,25 \mathrm{dBs}$}

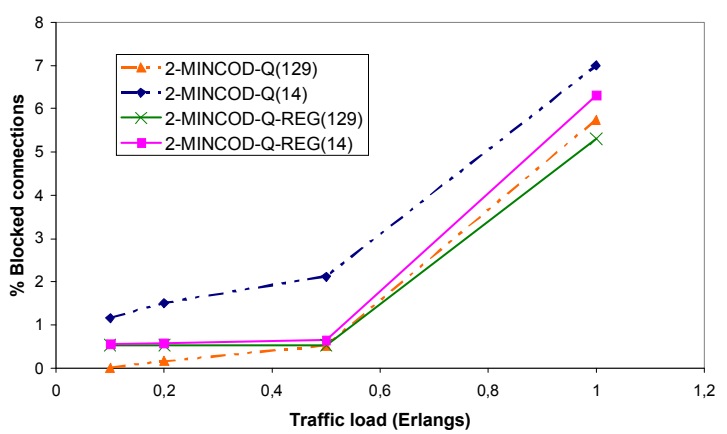

a)

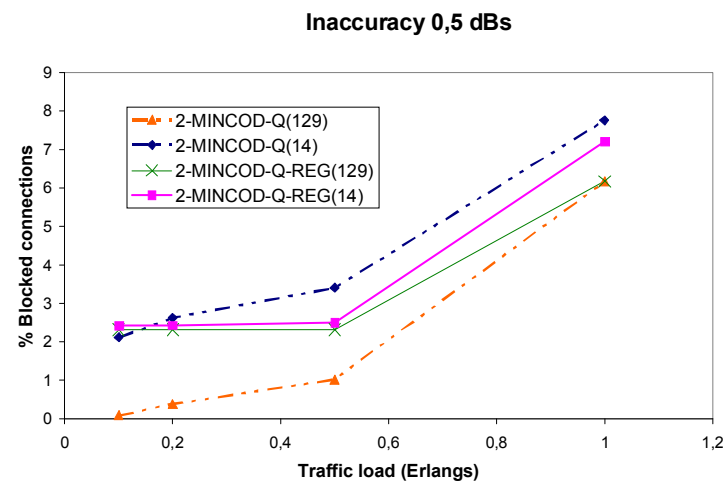

b)
Technology, vol. 23, no. 3, pp. 955-71, Mar. 2005.

[8] X. Yang and B. Ramamurthy, "Sparse regeneration in translucent wavelength-routed networks: architecture, network design and wavelength routing," Photonic Network Com.., vol. 10, no. 1, pp. 39-53, January 2005.

[9] G. Shen, and W.D. Grover., "Sparse placement of electronic switching node for low blocking in translucent optical networks" OSA J. Opt. Networking, vol. 1, no. 12, pp. 424-441, December 2002.

[10] R. Martínez, R. Casellas, R. Muñoz, T. Tsuritani and T.Otani, "Experimental GMPLS routing for dynamic provisioning in translucent wavelength switched optical networks", in Proc. OFC/NFOEC 2009, San Diego, USA, March 2009.

[11] A. Jukan, and G. Franzl, "Path Selection methods with multiple constraints in service-guaranteed WDM networks," IEEE/ACM Transactions on Networking vol.12, no. 1, pp. 59- 72, Feb. 2004

[12] M. Yannuzzi, M. Quagliotti, G. Maier, E. Marin-Tordera, X. MasipBruin, S. Sanchez-Lopez, J. Sole-Pareta, W. Erangoli, and G. Tamiri., "Performance of translucent optical networks under dynamic traffic and uncertain physical-layer information", ONDM 2009, Braunschweig, Germany, February 2009.

[13] F. Leplingard, A. Morea, T. Zami, N. Brogard, "Interest of an Adaptive Margin for the Quality of Transmission Estimation for Lightpath Establishment", OFC'2009, San Diego, California, March 2009.

[14] D. Pennickx and C. Perret, "Analysis of $10 \mathrm{~Gb} / \mathrm{s}$ Transparent Optical Networks", IEEE Photonics Technology Letters, Vol. 15, №5, May 2003.

[15] T. Zami, "Robustness of Quality estimators for IC-RWA to uneven channel powers in core networks", ICTON 2008, Athens, Greece, June 2008

[16] T. Zami, A. Morea, F. Leplingard and N. Brogard, "The relevant impact of the physical parameters uncertainties when dimensioning an optical core transparent network", ECOC 2008, Brussels, Belgium, September 2008.

[17] E. Marín, s. Sánchez, X. Masip,J. Solé, G. Maier, W. Erangoli, S.Santoni and M. Quagilotti, "MINCOD-MTD: A RWA Algorithm in Semi-Transparent Optical Networks", ECOC 2007, Rome, Italy, June 2007.

\section{Inaccuracy $\mathbf{0 , 2 5}$}

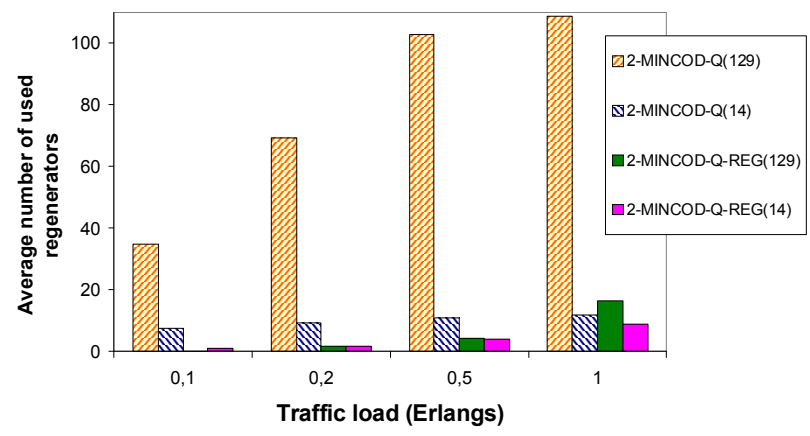

b)

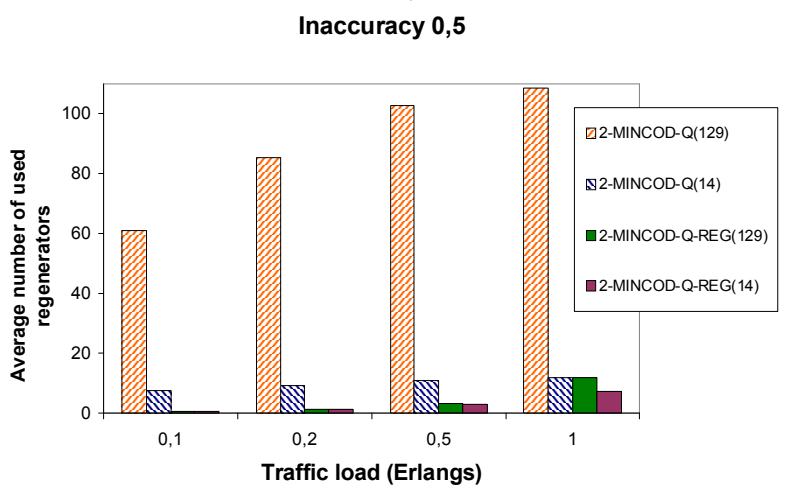

c)

Figure 3 Effect of the regenerator reduction with inaccurate $Q$ information 